\title{
Advances In Variable Pressure Imaging and Detection
}

\author{
Luyang Han $^{1}$, Christina Berger ${ }^{1}$, Markus Boese ${ }^{1}$, Alexander Thesen ${ }^{1}$, Fang Zhou ${ }^{1}$, Stefan Meyer ${ }^{1}$ and \\ Erik Essers $^{1}$ \\ 1. Carl-Zeiss Microscopy, FE-SEM Department, Oberkochen, Germany
}

The variable pressure scanning electron microscopy (VPSEM) has gained considerable interest since its commercial introduction more than 30 years ago [1]. Unlike a conventional SEM, which functions only in high vacuum, the VPSEM can be operated under a gas pressure in the specimen chamber. This permits observation of non-conductive samples without coating, as well as outgassing specimens, which are normally not compatible with high vacuum. However, due to the skirt effect [2] of the primary electron beam, resolution of a VPSEM is usually significantly reduced compared to a conventional SEM. Furthermore the VPSEM is also particularly troublesome for EDS applications, because additional $\mathrm{X}$-ray signal is produced by the electron skirt. In this contribution we will introduce the newly developed VP implementation of next-generation Zeiss SEM, which features not only a very restricted skirt effect under high gas pressures, but also enables the detection of pure secondary electron (SE) signal using all Zeiss Gemini ${ }^{\mathrm{TM}}$ technology in-lens detectors.

The essential component of our VP implementation is illustrated in Figure 1. It is composed of a coneshaped pressure limiting aperture called "beam sleeve" and a BSE detector, which are mounted on a pneumatically movable arm. This retractable VP module can be inserted pneumatically with high reproducibility. When inserted, the pressure-limiting aperture permits up to $500 \mathrm{~Pa}$ gas pressure in the specimen chamber, while the pressure inside the column and the objective lens is maintained at a much better vacuum. This enables the usage of the $8 \mathrm{kV}$ beam booster during VP operation, and thereby VP in-lens detection and high resolution at low beam energy [3]. The Zeiss InLens and EsB detectors can now detect SE and BSE signals in the VP mode thanks to the beam booster. This improves the SNR of the SE signal significantly, and it makes additionally available the low angle BSE signal. By a voltage of up to $400 \mathrm{~V}$, which can be applied to the pressure limiting aperture, secondary electrons are attracted by the aperture and directed to the InLens detector. For the detection of the high angle BSE signal, a 5segment diode detector is also integrated in the retractable VP module.

Three factors enable us to achieve high resolution in our VP implementation. First of all, the electron optic system is not influenced by the VP operation. This results in the same lens aberrations and the same beam spot size compared to the high vacuum mode. Secondly, the cone-shaped pressure limiting aperture helps to keep beam gas path length (BGPL) short. Even for EDS applications the BGPL is kept below $3 \mathrm{~mm}$, which significantly reduces the background X-ray signal. Lastly, the InLens detector detects the true SE signal, which carries only the surface information.

Figure 2 demonstrates some VP application images, which are taken with the retractable VP module. The left image shows natural fibers coated with Ag nanoparticles imaged at $80 \mathrm{~Pa}$ and $10 \mathrm{kV}$ at relative low magnification. The InLens detector and the EsB detector are used to obtain surface topography and material contrast, respectively, and these two kinds of contrast are clearly separated. The middle image shows a medium magnification of filter paper, imaged at $80 \mathrm{~Pa}$ and $3 \mathrm{kV}$. The charging effect is completely eliminated and an excellent SNR is obtained thanks to the InLens detector. The right image shows at a very high magnification an Au/Pd thin film deposited on glass. Although the $\mathrm{Au} / \mathrm{Pd}$ thin film 
is conductive, the film is not grounded but floating on an insulator. Imaging this sample in high vacuum will cause charge buildup and constant shifting of focus. In VP mode the fine grain structure smaller than $10 \mathrm{~nm}$ can be clearly resolved.

Overall, the new VP implementation permits operation at higher pressure, with high resolution, pure SE contrast and improved signal detection efficiency.

\section{References:}

[1] G. D. Danilatos, Scanning 3 (1980) p. 215.

[2] G. D. Danilatos, “Foundations of Environmental Scanning Electron Microscopy”, (Academic Press) p. 109.

[3] E. Essers, United States Patent US 6,590,210 B1
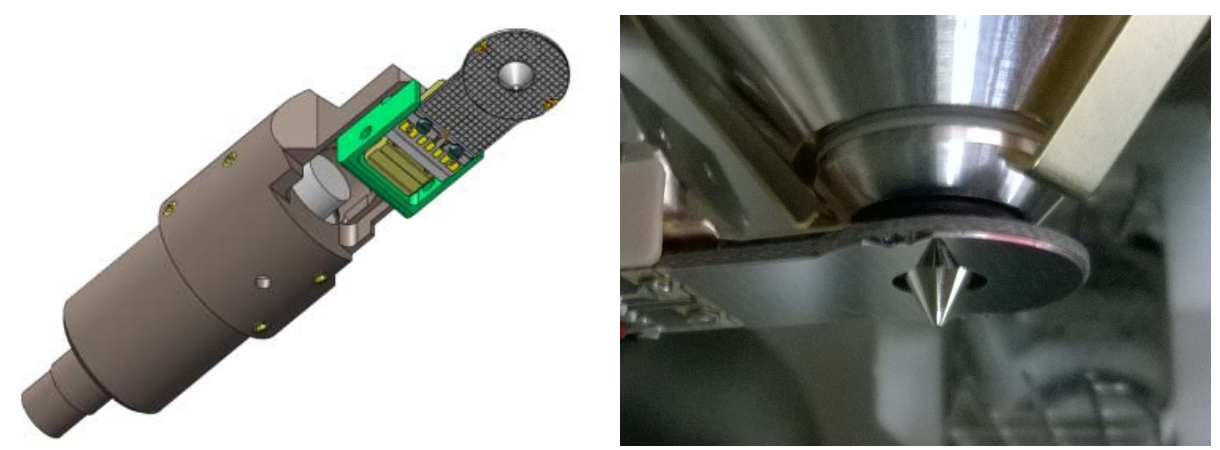

Figure 1. Pneumatically retractable VP module with a 5-segment BSE detector and the cone-shaped pressure limiting aperture. Left: Sketch Right: Photo of the inserted beam sleeve under the objective lens.
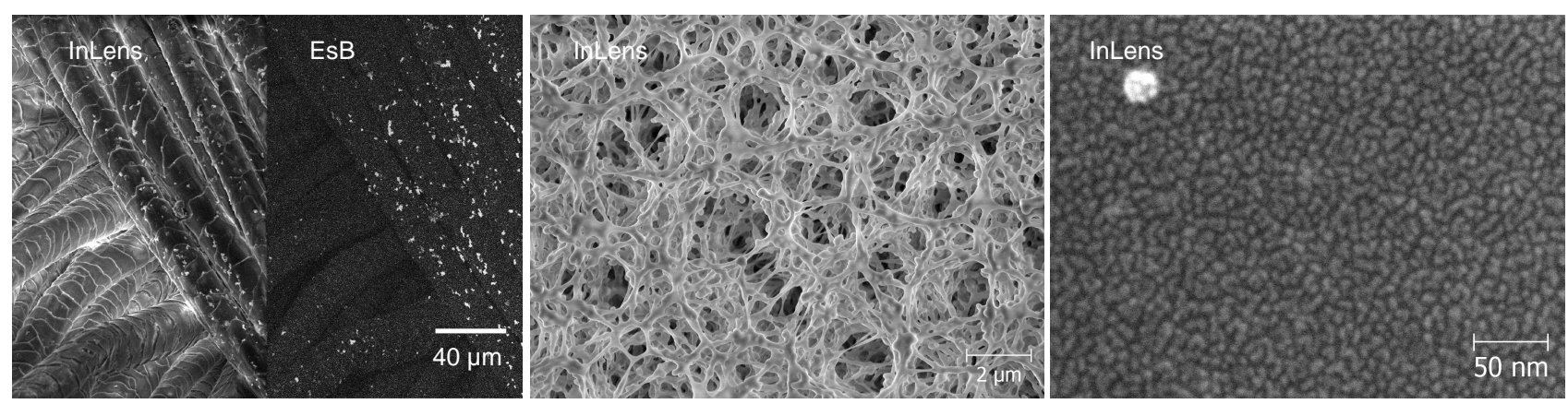

Figure 2. VP application examples imaged with the retractable VP module. Left: Ag nanoparticle coated natural fibers imaged with InLens and EsB detectors with low magnification at $80 \mathrm{~Pa}$ and $10 \mathrm{kV}$, sample courtesy of SBUK and Dr. Frank Simon, Leibniz-Institute for Polymer Research Dresden. Middle: Filter paper imaged with medium magnification at $80 \mathrm{~Pa}$ and $3 \mathrm{kV}$. Right: Au/Pd thin film deposit on glass imaged with high magnification at $40 \mathrm{~Pa}$ and $5 \mathrm{kV}$. 